\title{
Neonatal multi-modal cortical profiles predict 18-month
}

\section{developmental outcomes}

Daphna Fenchel ${ }^{1,2}$, Ralica Dimitrova ${ }^{3}$, Emma C. Robinson ${ }^{4}$, Dafnis Batalle ${ }^{2,3}$, Andrew Chew ${ }^{3}$, Shona Falconer ${ }^{3}$, Vanessa Kyriakopoulou ${ }^{3}$, Chiara Nosarti ${ }^{3,5}$, Jana Hutter ${ }^{3}$, Daan Christiaens ${ }^{3,6}$, Maximilian Pietsch ${ }^{3}$, Jakki Brandon ${ }^{3}$, Emer J. Hughes ${ }^{3}$, Joanna Allsop ${ }^{3}$, Camilla O'Keeffe ${ }^{3}$, Anthony N. Price ${ }^{3}$, Lucilio Cordero-Grande ${ }^{3,7}$, Andreas Schuh ${ }^{8}$, Antonios Makropoulos ${ }^{8}$, Jonathan Passerat-Palmbach ${ }^{8}$, Jelena Bozek ${ }^{9}$, Daniel Rueckert ${ }^{8,10}$, Joseph V. Hajnal ${ }^{3}$, Grainne McAlonan 1,2,11, A. David Edwards ${ }^{1,3}$, Jonathan O’Muircheartaigh ${ }^{1,2,3}$

1 MRC Centre for Neurodevelopmental Disorders, King's College London, London, SE1 1UL, UK

2 Sackler Institute for Translational Neurodevelopment, Department of Forensic and Neurodevelopmental Sciences, Institute of Psychiatry, Psychology \& Neuroscience, King's College London, London, SE5 8AF, UK

3 Centre for the Developing Brain, Department of Perinatal Imaging \& Health, School of Biomedical Engineering \& Imaging Sciences, King's College London, London, SE1 7EH UK

4 Department of Biomedical Engineering, School of Biomedical Engineering \& Imaging Sciences, King's College London, London, SE1 7EU, UK

5 Department of Child and Adolescent Psychiatry, Institute of Psychiatry, Psychology \& Neuroscience, King's College London, London, SE5 8AF, UK

6 Department of Electrical Engineering, ESAT/PSI, KU Leuven, Leuven, Belgium

7 Biomedical Image Technologies, ETSI Telecomunicación, Universidad Politécnica de Madrid \& CIBERBBN, Madrid, Spain

8 Biomedical Image Analysis Group, Department of Computing, Imperial College London, London, SW7 2AZ, UK

9 Faculty of Electrical Engineering and Computing, University of Zagreb, Zagreb, Croatia

10 Institute für Artificial Intelligence and Informatics in Medicine, Klinikum rechts der Isar, Technical University of Munich, Munich, Germany

11 South London and Maudsley NHS Foundation Trust, London, SE5 8AZ, UK

Corresponding author: Dr Jonathan O’Muircheartaigh, jonathanom@kcl.ac.uk, Forensic and Neurodevelopmental Sciences Department, Institute of Psychiatry, Psychology and Neuroscience, King's College London, 16 De Crespigny Park, London SE5 8AF 


\section{Abstract}

Developmental delays in infanthood often persist, turning into life-long difficulties, and coming at great cost for the individual and community. By examining the developing brain and its relation to developmental outcomes we can start to elucidate how the emergence of brain circuits is manifested in variability of infant motor, cognitive and behavioural capacities. In this study, we examined if cortical structural covariance at birth, indexing coordinated development, is related to later infant behaviour. We included 193 healthy term-born infants from the Developing Human Connectome Project (dHCP). An individual cortical connectivity matrix derived from morphological and microstructural features was computed for each subject (morphometric similarity networks, MSNs) and was used as input for prediction of behavioural scores at 18 months using Connectome-Based Predictive Modeling (CPM). Neonatal MSNs successfully predicted language and social-emotional performance. Predictive edges were distributed between and within known functional cortical divisions with a specific important role for primary and posterior cortical regions. These results reveal that multi-modal neonatal cortical profiles showing coordinated maturation are related to developmental outcomes and that network organization at birth provides an early infrastructure for future functional skills.

Keywords: perinatal; neonatal neuroimaging; infant development; morphometric similarity networks; brain development 


\section{Introduction}

Developmental delays occur in around 13\% of infants in the US population (Rosenberg et al. 2008). Delays can be observed in motor, cognitive, language and communicative domains, and when they persist, they are termed developmental disabilities. These can place a great emotional and financial burden on the individual, their family, and the general community (Shahat \& Greco, 2021; Stabile \& Allin, 2012). While some infants catch up with their peers, others will continue to present difficulties (Riva et al. 2021). Behavioural delays are associated with a higher likelihood of autism spectrum conditions (ASC), attention deficit hyperactivity disorder (ADHD) and schizophrenia (Gurevitz, Geva, Varon, \& Leitner, 2014; Landa \& Garrett-Mayer, 2006; Sorensen et al., 2010). Within the general population, developmental milestones are related to cognitive functions both in childhood and adulthood (Flensborg-Madsen \& Mortensen, 2018; Murray et al. 2007), reiterating their importance over the lifespan.

Risk factors for poor neurodevelopmental outcomes include familial history of neurodevelopmental conditions (Ozonoff et al. 2011; Shivers et al. 2019; Stromswold, 1998) and inherited or de novo genetic changes (Cooper et al. 2011; Marshall et al. 2008), in addition to preterm birth, low birth weight (Aylward, 2014; Pascal et al. 2018) and other perinatal complications (Mwaniki et al. 2012). However, most infants will have no recognized predisposing factor. Adding to this complexity, the pace of motor and language development in the first years of life is variable within and between individuals even within the 'normal' range (Fenson et al. 1994; Piek, 2002). Identifying individuals at potentially greater likelihood for difficulties allows for early interventions which have been found to improve outcome (Dawson et al. 2010; Jeong et al. 2021). Specifically, by being able to recognize babies in the general population who might need extra support, we can begin to address difficulties or potential difficulties very early on, while brain development is still in its early sensitive period (Reh et al. 2020). 
Prospectively profiling the developing brain and investigating its relationship with adaptive and maladaptive behaviours, promotes our understanding of innate and external factors contributing to variability, vulnerability, and resilience to adverse outcomes. MRI studies have concluded that structural and functional brain networks start to develop in the fetal period and continue to fine-tune during childhood (Batalle et al. 2018). However how (and when) this emerging brain architecture relates to behavioural outcomes in infanthood is yet to be determined. The majority of early developmental studies have focused on brain-behaviour relationship in preterm neonates, a group that on average has a greater likelihood of delayed or atypical development (Van't Hooft et al. 2015). More recently, a conceptual shift attempts to move from association to prediction (Rosenberg et al. 2018), with more studies examining brain structure in the term-born neonatal population with no apparent risks for a poorer developmental outcome (Girault et al. 2019b; Wee et al. 2017). Most attention has been given to the predictive ability of white matter connectivity (Ball et al. 2015; Girault et al. 2019a, 2019b; Keunen et al. 2017; Wee et al. 2017).

Morphometric similarity networks (MSNs) (Seidlitz et al. 2018) are based on structural covariance of brain regions whereby similarity between brain regions is thought to reflect synchronized maturation and relatedness (Alexander-Bloch et al. 2013a, 2013b). This has implications for efficient information transfer and functionality and therefore could potentially serve as a marker for the development of mental and motor abilities. MSNs incorporate multiple MRI modalities, both microstructural and morphological, to overcome the limitations of using individual features with particular spatiotemporal trajectories and provide a more comprehensive description of the brain, improving the predictive ability of clinical symptoms and behaviour from brain data (Liu et al. 2015; Tulay et al. 2019). In adults, MSNs are related to cognitive abilities and the expression of genes associated with neurodevelopmental conditions (Morgan et al. 2019; Seidlitz et al. 2018,2020).

In our previous work, we used this method to characterize the developing brain at neonatal timepoint using structural and diffusion indices (Fenchel et al. 2020), reporting a community structure largely 
aligned with known functional distinctions and network temporal trajectories, and showing close similarity with cytoarchitectural features (Ball et al. 2020).

In this current study, we are interested in furthering our understanding of how this neonatal cortical organization relates to infant developmental outcomes. Therefore, here we asked whether cortical profiles at term-birth, derived from MSNs, are associated with- and predictive of- motor, cognitive, language and social-emotional abilities at 18 months. We attempted to predict infant behaviour from neonatal MSNs using connectome-based predictive modelling (CPM), a data-driven linear approach to predict continuous measures of behaviours from individual connectivity matrices (Shen et al. 2017). Following CPM, we examined if network-strength summary measures at the whole cortex level and within cortical functional clusters were able to capture the same brain-behaviour patterns observed at the single-edges level. 


\section{Methods}

\subsection{Subjects}

This study included a sample of term-born healthy neonates participating in the Developing Human Connectome Project (dHCP); (http://www.developingconnectome.org/), scanned at the Newborn Imaging Centre at Evelina London Children's Hospital, London, UK. Images are openly available on the project website. This project has received ethical approval (14/LO/1169), and written informed consent was obtained from parents. As part of the dHCP project, subjects are invited for a follow-up visit to assess infant development at 18 months. This assessment includes The Bayley Scales of Infant and Toddler Development (Bayley-III) (BSID) (Bayley, 2006) exploring overall developmental aspects, as well as the Quantitative Checklist for Autism in Toddlers (Q-CHAT) (Allison et al. 2008) for assessment of social-emotional development. Out of the 241 subjects included in the initial analysis and for which MSNs were constructed (Fenchel et al. 2020), n=204 completed the Bayley-III assessment and $\mathrm{n}=198$ completed the Q-CHAT assessment. Only subjects with information on a proxy of socio-economic status, the Index of Multiple Deprivation (IMD) (https://tools.npeu.ox.ac.uk/imd/), were included to control for its possible confounding effect. This resulted in a sample size of $n=193$ with Bayley-III data and $n=187$ with Q-CHAT data. This resulted in a sample size of $n=193$ with Bayley-III data and $n=187$ with Q-CHAT data.

\subsection{Image acquisition and processing}

Neonatal MR brain images were acquired on a 3T Philips Achieva scanner without sedation, using a dedicated 32-channels head coil system (Hughes et al. 2017). Acquisition, reconstruction and processing of structural and diffusion images followed optimized protocols for the neonatal brain implemented as part of the dHCP pipeline and have been previously described in Fenchel et al. 2020. T2-weighted (T2w) images were obtained using a turbo spin echo (TSE) sequence, acquired in sagittal and axial planes with TR=12s, TE=156ms, SENSE factor 2.11 (axial) and 2.58 (sagittal) with overlapping slices (resolution $0.8 \times 0.8 \times 1.6 \mathrm{~mm})$. T1-weighted $(\mathrm{T} 1 \mathrm{w})$ images were acquired using an Inversion Recovery TSE sequence with the same resolution using TR=4.8s, TE=8.7ms, SENSE factor 
2.26 (axial) and 2.66 (sagittal). Structural images were reconstructed to a final resolution of $0.5 \times 0.5 \times 0.5 \mathrm{~mm}$, using slice-to-volume registration (Cordero-Grande et al. 2018). Structural processing followed the pipeline described in (Makropoulos et al. 2018): Motion- and bias-corrected $\mathrm{T} 2 \mathrm{w}$ images were brain extracted and segmented. White, pial and midthickness surfaces were genereated, inflated and projected onto a sphere. Brains were aligned to the 40 -week dHCP surface template (Bozek et al. 2018) using Multimodal Surface Matching (MSM) (Robinson et al. 2013, 2014). Cortical features including cortical thickness (CT), pial surface area (SA), mean curvature (MC), and the $\mathrm{T} 1 \mathrm{w} / \mathrm{T} 2 \mathrm{w}$ ratio images indicative of myelin content (MI) were extracted for each subject (Makropoulos et al. 2018).

Diffusion images were obtained using parameters $\mathrm{TR}=3.8 \mathrm{~s}$, TE=90ms, SENSE factor=1.2, multiband $=4$, partial Fourier factor=0.86, resolution $1.5 \times 1.5 \times 3.0 \mathrm{~mm}$ with $1.5 \mathrm{~mm}$ overlap (Hutter et al. 2018). Diffusion gradient encoding included images collected at $\mathrm{b}=0 \mathrm{~s} / \mathrm{mm}^{2}$ (20 repeats), $\mathrm{b}=400 \mathrm{~s} / \mathrm{mm}^{2}$ (64 directions), $\mathrm{b}=1000 \mathrm{~s} / \mathrm{mm}^{2}$ ( 88 directions), $\mathrm{b}=2600 \mathrm{~s} / \mathrm{mm}^{2}$ (128 directions) (Tournier et al. 2020). Diffusion images were denoised (Veraart et al. 2016), Gibbs-ringing suppressed (Kellner et al. 2016), and the field map estimated (Andersson et al. 2003). Images were corrected for subject motion and image distortion with slice-to-volume reconstruction using multi-shell spherical harmonics and radial decomposition (SHARD) and were reconstructed to a final resolution of $1.5 \times 1.5 \times 1.5 \mathrm{~mm}$ (Christiaens et al. 2021). A tensor model was fitted using a single shell $\left(\mathrm{b}=1000 \mathrm{~s} / \mathrm{mm}^{2}\right)$, and fractional anisotropy (FA) and mean diffusivity (MD) maps were generated using MRtrix3 (Tournier et al. 2019). Neurite density index (NDI) and orientation dispersion index (ODI) maps were calculated using the default NODDI toolbox implementation with default values (Zhang et al. 2012). Diffusion maps were registered onto individual T2w images using FSL's epi_reg (FLIRT) (https://fsl.fmrib.ox.ac.uk), and then projected onto the cortical surface using Connectome Workbench. All images were visually inspected for motion or image artefacts and data excluded accordingly (Fenchel et al. 2020), and images were checked for registration errors. 


\subsection{MSNs construction}

MSN construction for this cohort was described previously in Fenchel et al. 2020 and summarized in Figure1. Briefly, the cortical surface was parcellated into 75 bilateral equal-sized regions with Voronoi decomposition. Seven of these regions were excluded due to diffusion signal dropout. Each region was then characterized by an eight-feature vector of mean normalized values of four structural features: CT, MC, MI and SA and four diffusion features: FA, MD, NDI and ODI. Pearson's correlation between the eight-feature vector for every pair of regions was calculated, resulting in a 143 x 143 similarity-based connectivity matrix for each subject. Values were Fisher's-z-transformed before analysis.

\subsection{Behavioural developmental assessment}

Mean age at developmental assessment was 18.69 \pm 1.04 months (range 17.16-24.46 months), mean corrected age for gestational age (GA) at birth was 18.68 \pm 1.00 months (range 17.30-24.33 months), the latter used for Bayley-III score calculation. The Bayley-III test is a commonly used tool for tracking infants' development, targeting to identify possible developmental delays. Standardized scores are divided into a motor composite score, derived from gross (GM) and fine (FM) motor scaled sub-scales, a language composite score derived from expressive (EL) and receptive scaled sub-scales (RL) and a cognitive composite score. Bayley-III assessments were completed by trained practitioners. A higher score on these scales reflects better performance.

Social-emotional development was determined by the Q-CHAT, a parent-based report of 25 items including joint attention, pretend play, language development, repetitive behaviours, and social communication. A higher summary score suggests more social-emotional difficulties.

\subsection{Prediction of developmental outcomes from MSNs}

We utilized Connectome-based Predictive Modeling (CPM) (Shen et al. 2017) to explore the predictive ability of neonatal MSNs for four developmental outcomes measures, the three Bayley-III composite scores (cognitive, language and motor) and the Q-CHAT score. The model is trained each time on $\mathrm{n}-1$ subjects and is tested on the left-out subject for calculation of the predicted behavioural 
scores (leave-one-out cross validation). Each individual edge was partially correlated (Spearman's r) with the behavioural measure, controlling for postmenstrual age (PMA) at scan, sex, total intracranial volume (ICV), IMD and time from birth to scan. Positive edges (associated with higher behavioural scores) and negative edges (associated with lower behavioural scores) with $\mathrm{p}<0.05$ were then selected. For each subject in the training set, these edges are summed separately to create a positive network sum and a negative network sum. Linear regression with no intercept (Rosenberg et al. 2018; Shen et al. 2017) linking the positive and negative sums was then preformed. The predicted behaviour for the left-out subject is calculated by fitting this subject's sum of positive and negative edges identified in the training set, adjusted for covariates, with the beta coefficients derived from the training model. Model performance was assessed by computing Spearman's $r$ between the observed and predicted behaviours. This performance was assessed by generating a null distribution of $r$ values from $\mathrm{N}=999$ random permutations of the behavioural data. The resulting $p$-value is calculated as the number of $r$ values equal or larger to the original $r$ value divided by $\mathrm{N}+1$.

Predictive networks were defined by taking edges appearing in at least $90 \%$ of testing runs. For clarity and ease of interpretation, predictive edges were examined in the context of the seven clusters reported before in Fenchel et al. (2020): occipital \& parietal, limbic, anterior frontal, insular \& medial frontal, fronto-temporal, cingulate and somatosensory \&auditory. For each cluster, we (1) summed separately the number of predictive positive and negative edges within the cluster and divided that by the number of all possible edges within that cluster to control for cluster size and (2) summed separately the number of positive and negative edges between each pair of clusters and divided that by the number of all possible edges between those pairs.

\subsection{Association between MSNs summary measures and developmental measures}

The association between whole-network average strength (across the entire cortex) and the eight developmental measures was examined by averaging a symmetric triangle of the connectivity matrix, excluding self-connections. This was entered together with PMA at scan, sex, total intracranial volume, IMD and time from birth to scan into a general linear model where the developmental measure was the dependant variable. Partial $\mathrm{R}^{2}$ for the brain network measure was calculated as: (SSE 
bioRxiv preprint doi: https://doi.org/10.1101/2021.09.23.461464; this version posted September 24, 2021. The copyright holder for this preprint (which was not certified by peer review) is the author/funder, who has granted bioRxiv a license to display the preprint in perpetuity. It is made available under aCC-BY-NC 4.0 International license.

reduced model-SSE full model)/SSE reduced model. Although PMA at scan, ICV and time from birth to scan were significantly correlated $(\mathrm{r}=0.71, \mathrm{p}<0.001)$, no variance inflation factor (VIF) exceeded 5

(Craney \& Surles, 2002) and therefore we retained all covariates for all analyses.

a. Parcellate cortex inco approx, equally sized regions (Voronoi)

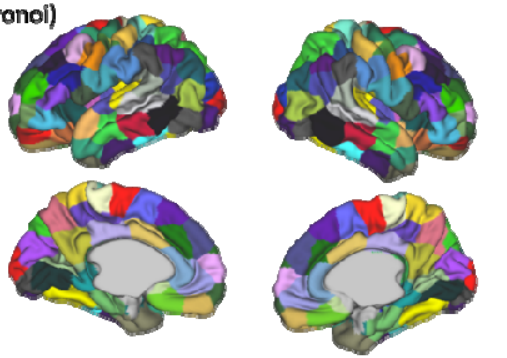

C. Calculate inter-reglonal simllarity (Pearson's $r$ )

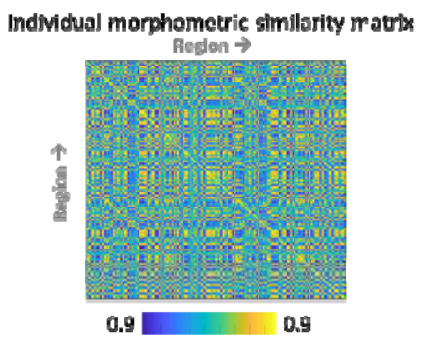

b. Extract average morphological and microstructural measures per region and subject

\begin{tabular}{|c|c|c|c|c|c|c|c|}
\hline \multicolumn{3}{|c|}{ Anatomical Surface Metrics } & \multicolumn{2}{|c|}{ Tensor Metrics } & \multicolumn{2}{|c|}{ NODDI Metrics } \\
\hline CT & SA & MI & MC & FA & MD & NDI & ODI \\
\hline
\end{tabular}

d. Link with 18-month behavioural data

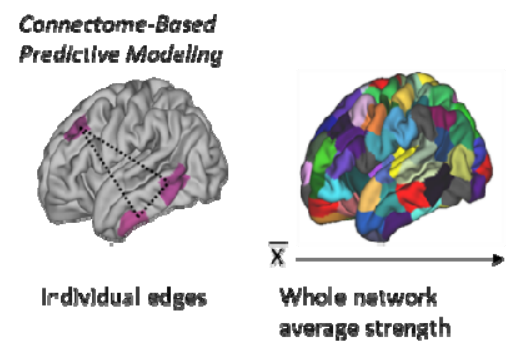

Figure 1. Pipeline Morphometric Similarity Networks construction and behavioural analysis.

a. Regions are defined using Voronoi tessellation of the cortical surface; $b$. A feature vector of averaged normalized values of cortical thickness (CT), mean curvature (MC), myelin index (MI), surface area (SA), fractional anisotropy (FA), mean diffusivity (MD), neurite density index (NDI) and orientation dispersion index (ODI) is derived for each region; c. Each pair of regions is correlated using Pearson's r, resulting in an individual similarity-based connectivity matrix. d. Network strength at a whole-network level and single-edges level is related to behavioural measures by means of association and prediction respectively. 


\section{Results}

\subsection{Demographics and behavioural scores}

Demographics of the sample and mean behavioural scores are presented in Table 1. All Bayley-III items were positively correlated with each other and negatively correlated with the Q-CHAT (Supplementary Table 1). Corrected age at assessment was not associated with either the Bayley-III scores or the Q-CHAT score.

Table 1. Demographics and behavioural scores

\begin{tabular}{|c|c|c|}
\hline \multicolumn{1}{|c|}{} & N(\%)/Median(range) \\
\hline \multirow{4}{*}{ Demographics } & Sex (male) & $101(52.3 \%)$ \\
\cline { 2 - 3 } & GA at birth (weeks) & $40.14(37.29-42.14)$ \\
\cline { 2 - 3 } & PMA at scan (weeks) & $40.86(37.43-44.43)$ \\
\cline { 2 - 3 } & Time from birth to scan (weeks) & $0.29(0-5.28)$ \\
\hline \multirow{4}{*}{ Bayley-III } & IMD & $26.12(1.55-61.37)$ \\
\cline { 2 - 3 } & & Mean \pm SD \\
\cline { 2 - 3 } & Cognitive composite & $99.95 \pm 10.19$ \\
\cline { 2 - 3 } & Language composite & $96.39 \pm 15.41$ \\
\cline { 2 - 3 } & EL sub-scale & $8.81 \pm 2.58$ \\
\cline { 2 - 3 } & RL sub-scale & $9.90 \pm 3.14$ \\
\cline { 2 - 3 } & Motor composite & $101.41 \pm 9.70$ \\
\hline Q-CHAT & FM sub-scale & $11.39 \pm 2.20$ \\
\hline
\end{tabular}

GA- gestational age, PMA- postmenstrual age, IMD- Index of Multiple Deprivation, Bayley-IIIBayley Scales of Infant and Toddler Development, EL- expressive language, RL- receptive language, FM- fine motor, GM- gross motor, Q-CHAT- Quantitative Checklist for Autism in Toddlers

\subsection{Single edges prediction- CPM}

Neonatal MSNs successfully predicted the language composite scores $\left(r_{\mathrm{s}}=0.196, \mathrm{p}=0.007, \mathrm{p}\right.$ permute $=0.019)$ and the Q-CHAT score $\left(r_{\mathrm{s}}=0.182, \mathrm{p}=0.011, \mathrm{p}\right.$ permute $\left.=0.024\right)$ (Figure 2). The positive and negative predictive networks for the Q-CHAT included $1.66 \%$ and $2.80 \%$ of all possible connections, respectively. The positive and negative predictive networks for language included $1.80 \%$ and $1.55 \%$ of all possible connections, respectively. The proportion of edges within each of the seven clusters included in the predictive networks are presented in Figure 3. For Q-CHAT, the highest proportion of positive predictive edges was within the anterior frontal and occipital and parietal 
clusters, while the cingulate did not show any within-cluster predictive edges. For the negative predictive network however, the highest proportion of edges was observed in the cingulate cluster. The insular and medial frontal showed the highest proportion of positive predictive edges for language scores. The negative language network only involved two within-clusters edges, namely the fronto-temporal and anterior frontal clusters. Limbic edges were not involved in any of the four predictive networks.
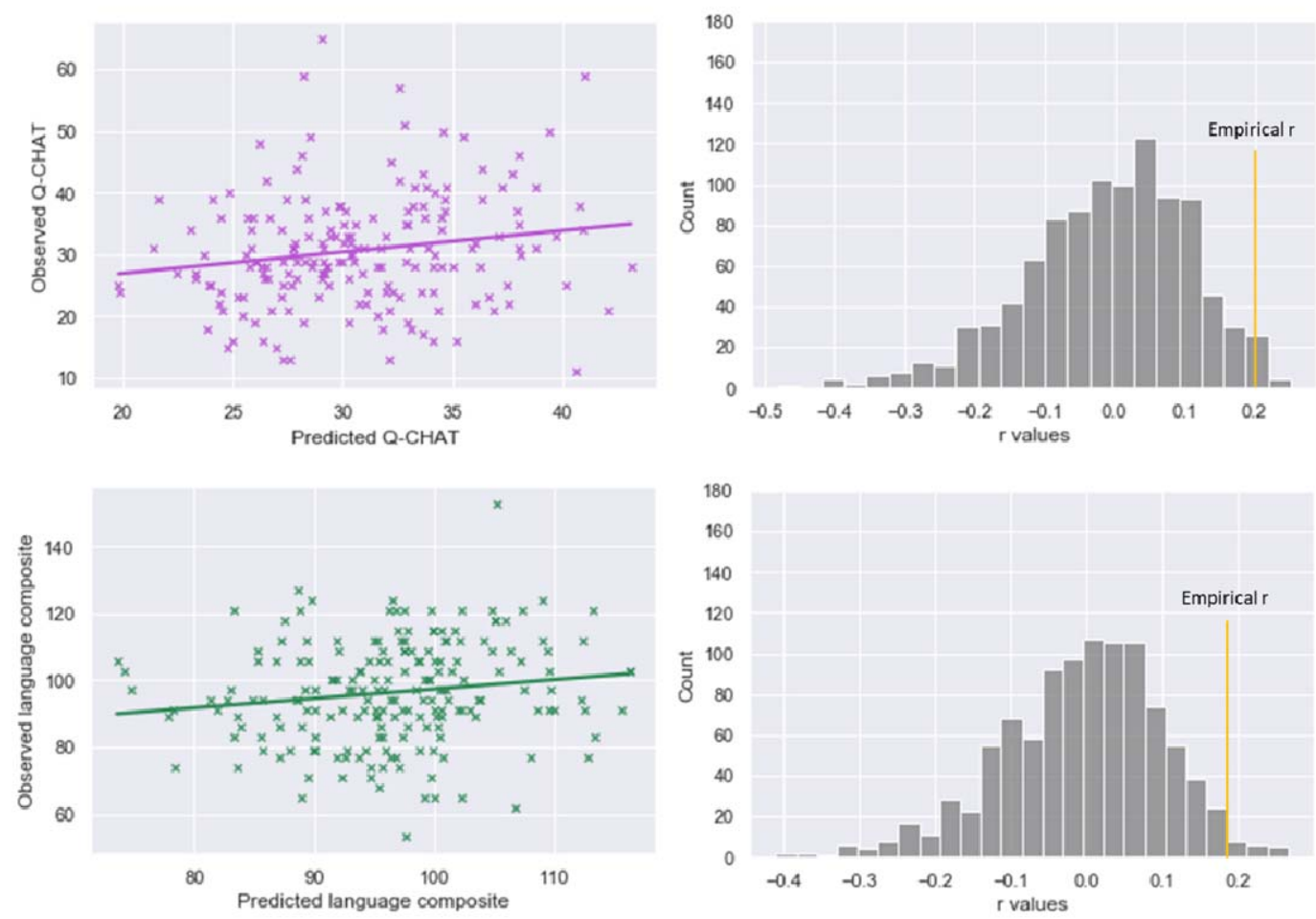

Figure 2. Prediction of Language and Q-CHAT scores from neonatal MSNs using Connectomebased Predictive Modeling (CPM).

Plots of significant correlation between predicted and observed language and Q-CHAT scores (left) and results of null r values with permutation testing (right) using Connectome-based Predictive Modeling (CPM). 


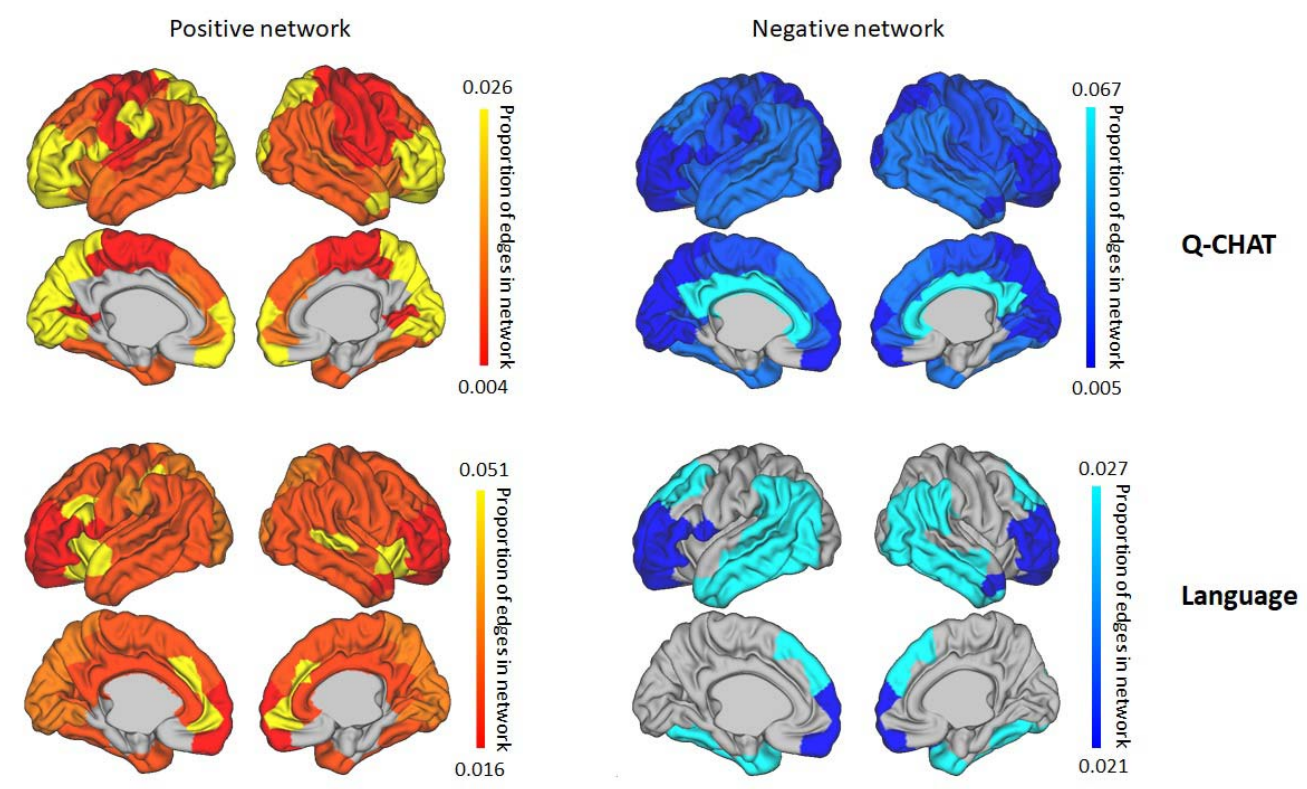

Figure 3. Proportion of within-cluster edges involved in predictive networks.

Proportion of edges included in successful prediction models connecting nodes within each of the seven clusters.

The proportion of predictive edges connecting between clusters are presented in Figures 4 and 5 . While the relationship emerging between clusters is complex, two important observations are made: First, the highest proportion of predictive edges involved the occipital and parietal cluster in all four networks. Second, the positive network of the Q-CHAT was similar to the negative network of the language composite, and the negative network of the Q-CHAT was similar to the positive network of the language composite. Therefore we looked at the overlap between these networks and found that $16.6 \%$ of edges in the negative language network overlapped with the positive Q-CHAT network and $26.8 \%$ of edges in the positive language network overlapped with the negative Q-CHAT network. No overlapping edges were found between the two positive networks or the two negative networks. 


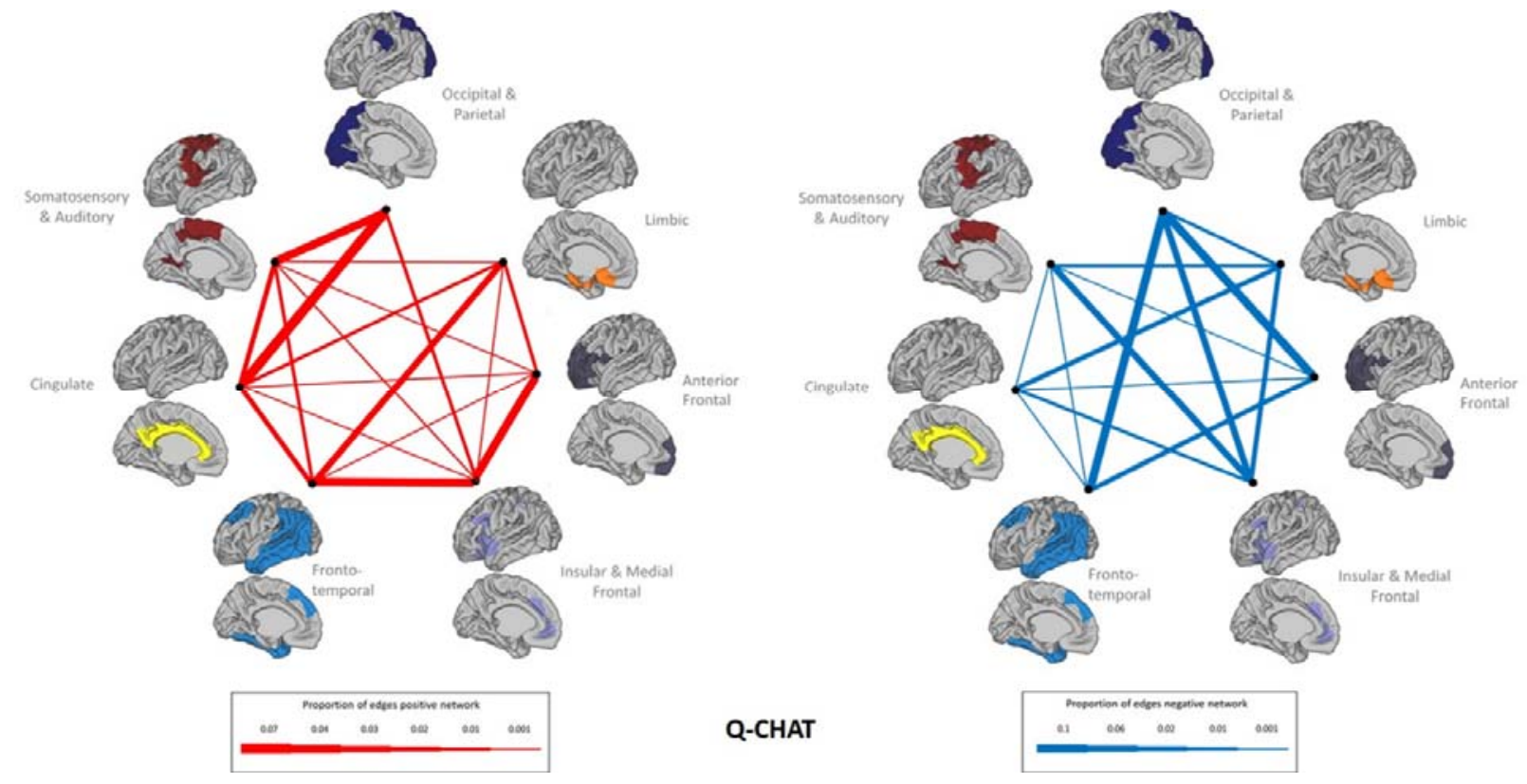

Figure 4. Proportion of between-cluster edges involved in Q-CHAT predictive networks. Proportion of edges included in Q-CHAT prediction model connecting nodes between clusters.

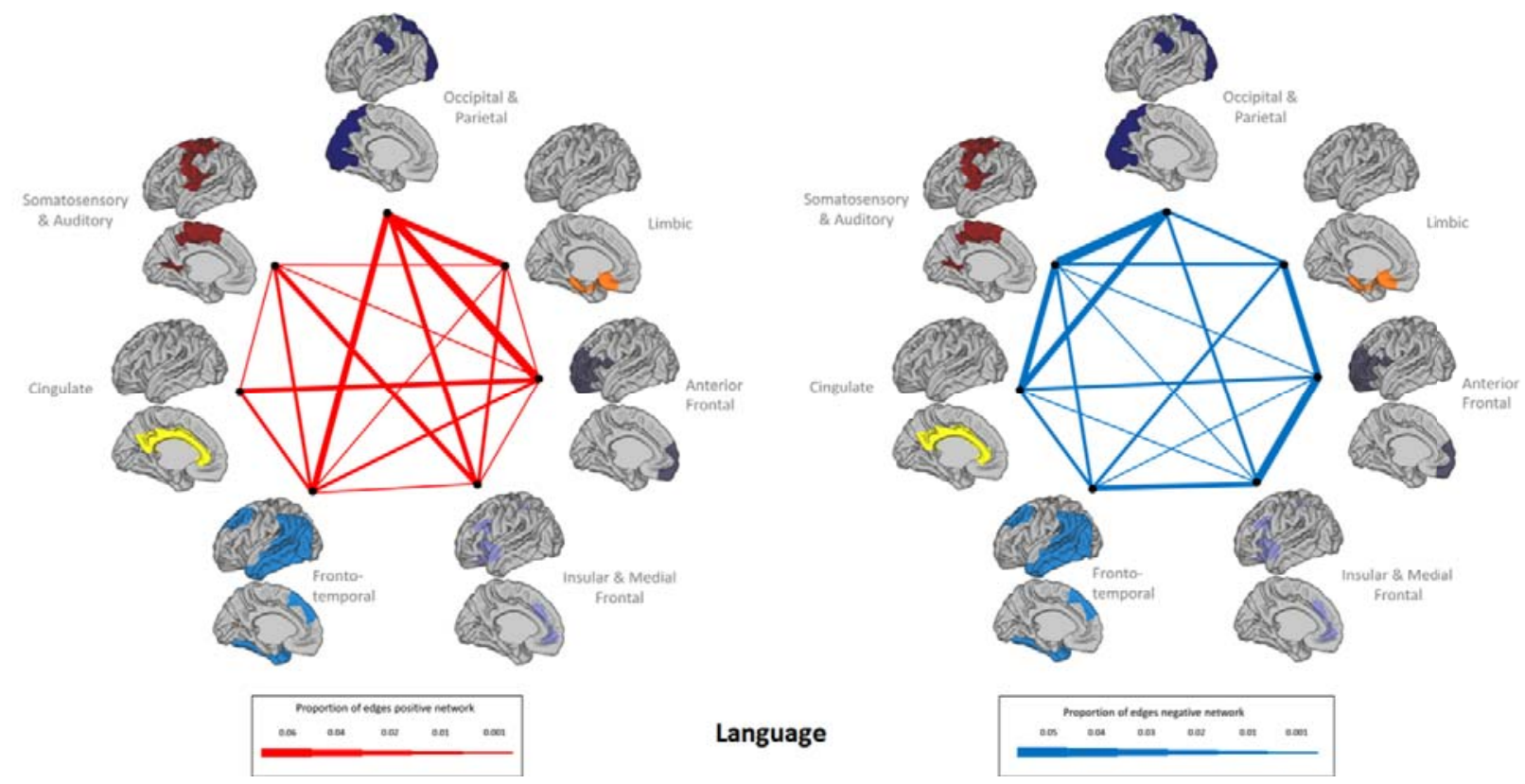

Figure 5. Proportion of between-cluster edges involved in language predictive networks.

Proportion of edges included in language prediction model connecting nodes between clusters. 


\subsection{Whole-network average strength}

There were significant positive associations between whole-network average strength and language composite score and EL sub-score, and a negative significant association with the Q-CHAT scores. No associations were found for the motor composite and sub-scales or the cognitive composite (Figure 6, Table 2). Variance explained by the full model was $7 \%$ for EL, $10 \%$ for language and $14 \%$ for Q-CHAT, with specific contribution of network strength estimated at 4\%,3\% and $4 \%$ respectively $\left(\right.$ partial $\left.\mathrm{R}^{2}\right)$.
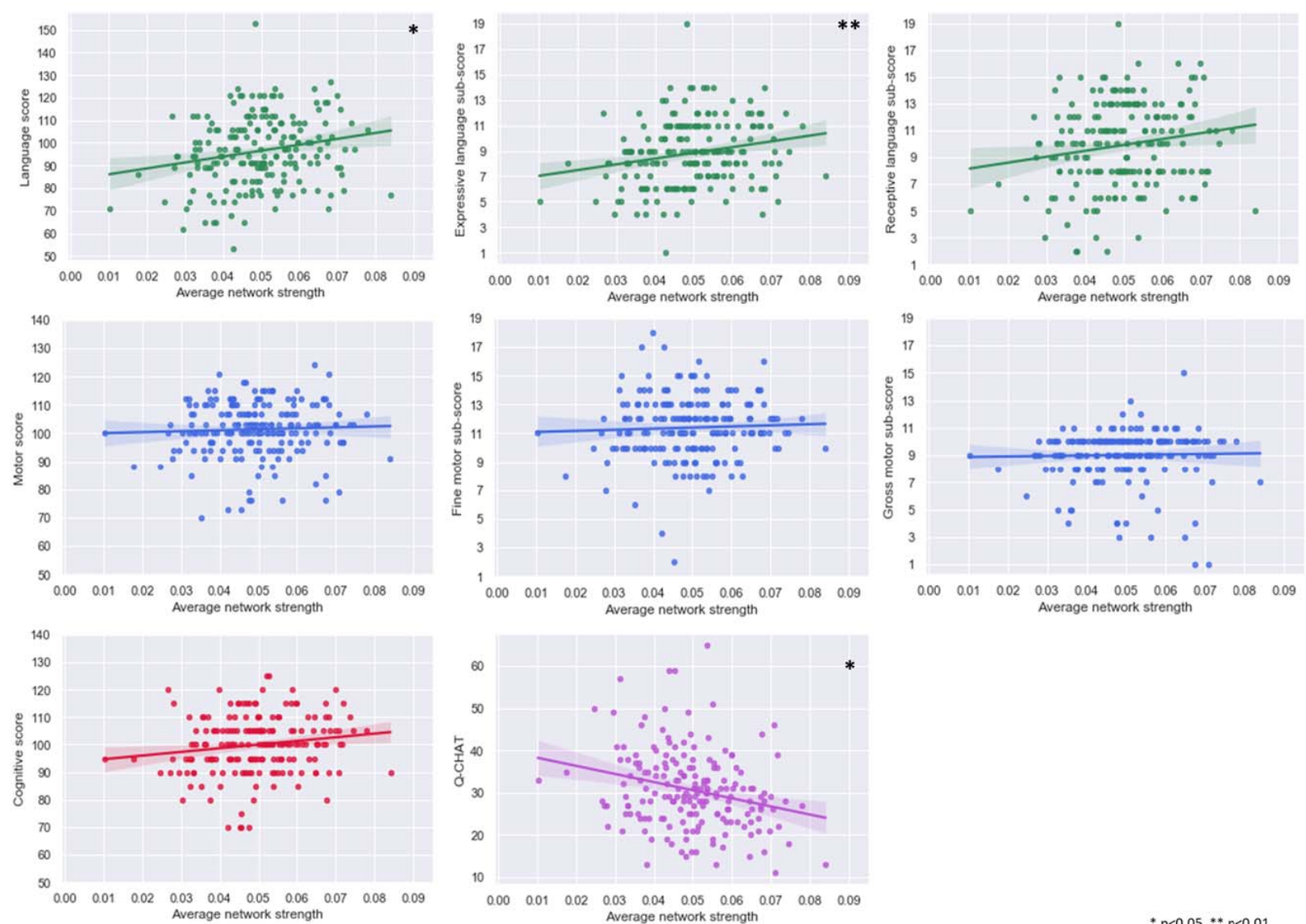

${ }^{*} p<0.05,{ }^{* *} p<0.01$

Figure 6. Scatterplots whole-network average and behaviour.

Plots of average network strength across the cortex and behavioural measures at 18 months. 
Table 2. Linear regression results for whole-network average strength and behaviour

\begin{tabular}{|c|c|c|c|c|}
\hline & Beta $(\mathbf{9 5 \%} \mathbf{C I})$ & SE & T-value & $\begin{array}{c}\mathbf{R}^{2} \text { full } \\
\text { ANOVA model }\end{array}$ \\
\hline Cognitive composite & $114.78(-19.19-248.75)$ & 67.91 & 1.69 & 0.07 \\
\hline Language composite & $221.91(23.14-420.67)$ & 100.75 & $2.20 *$ & 0.10 \\
\hline EL sub-scale & $45.58(11.67-79.49)$ & 17.90 & $2.65 * *$ & 0.07 \\
\hline RL sub-scale & $31.02(-9.32-71.36)$ & 20.45 & 1.52 & 0.11 \\
\hline Motor composite & $39.97(-91.57-171.51)$ & 66.68 & 0.6 & 0.01 \\
\hline FM sub-scale & $6.0(-23.50-35.50)$ & 14.95 & 0.4 & 0.03 \\
\hline GM sub-scale & $7.19(-18.73-33.10)$ & 13.14 & 0.55 & 0.02 \\
\hline Q-CHAT & $-153.78(-271.31-(-36.26))$ & 59.56 & $-2.58 *$ & 0.14 \\
\hline
\end{tabular}

$* \mathrm{p}<0.05, * * \mathrm{p}<0.01$; SE-Standard error, EL- expressive language, RL- receptive language, FM- fine motor, GM- gross motor, Q-CHAT- Quantitative Checklist for Autism in Toddlers.

To elucidate whether network strength is valuable in the context of the community structure (clusters) of neonatal MSNs reported previously (Fenchel et al. 2020), we examined the average network strength within each of the seven clusters. The network strength within the insular and medial frontal cluster was related to scores on the language composite $\left(\beta=25.82,95 \% \mathrm{CI}=3.30-48.33, \mathrm{p}<0.05, \mathrm{R}^{2}\right.$ full $=0.11, R^{2}$ partial=0.03), RL sub-scale $\left(\beta=5.37,95 \% \mathrm{CI}=0.84-9.91, \mathrm{p}<0.05, \mathrm{R}^{2}\right.$ full $=0.13, \mathrm{R}^{2}$ partial=0.03), cognitive composite $\left(\beta=15.44,95 \% \mathrm{CI}=0.30-30.58, \mathrm{p}<0.05, \quad \mathrm{R}^{2}\right.$ full $=0.08, \mathrm{R}^{2}$ partial=0.02), and Q-CHAT ( $\beta=-14.86,95 \% \mathrm{CI}=-28.45-(-1.26), \mathrm{p}<0.05, \mathrm{R}^{2}$ full=0.13, $\mathrm{R}^{2}$ partial=0.03), and within the somatosensory and auditory cluster to language composite $(\beta=18.94,95 \%$ CI=1.3936.50, $\mathrm{p}<0.05, \mathrm{R}^{2}$ full $=0.10, \mathrm{R}^{2}$ partial=0.02) and EL sub-scale $(\beta=3.97,95 \% \mathrm{CI}=0.97-6.96, \mathrm{p}<0.01$, $\mathrm{R}^{2}$ full=0.07, $\mathrm{R}^{2}$ partial=0.04) (Figure 7). 

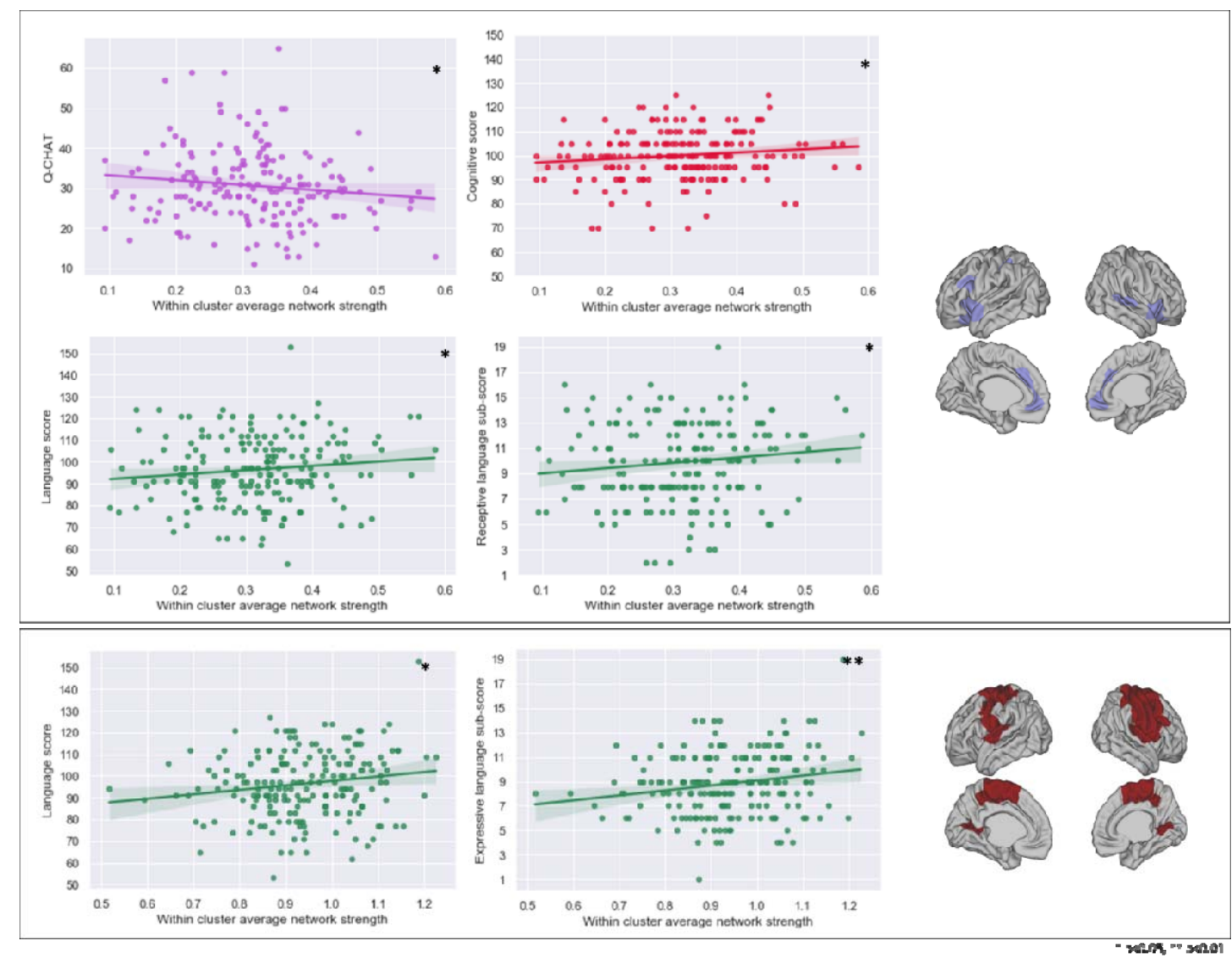

Figure 7. Scatterplots of significant associations of within-cluster average network strength and behaviour.

Plots of significant associations between within cluster average network strength and behavioural measures at 18 months. Top: insular \& medial frontal cluster, bottom: somatosensory \& auditory cluster. 


\section{Discussion}

In this study, we found that neonatal MSNs can successfully predict language abilities and socialemotional behaviours at 18 months in a large group of healthy term-born babies. We show that the pattern of cortical maturation at birth already captures variability in infant development. This association between brain structure at birth and infant developmental outcomes was not limited to information held in individual edges of the network but was also evident in summary measures of network strength over the entire cortex and specifically in somatosensory-auditory, insular and medial frontal areas. On a regional level, the predictive networks were complex and widespread across the cortex, albeit suggesting specific important involvement of primary and posterior cortical regions. No consistent or significant results were found for the cognitive or motor measures.

Both language and social-emotional development is thought to result from a combination of fetal and postnatal brain programming, together with in- and ex-utero experiences. However exactly how these factors come about and interact to create these complex behaviours is still under investigation. Our results indicate that some of the neural foundations crucial for developmental capacities in infanthood, observed through the pattern of structural covariance, originate during the fetal period and are already present at birth. Language and social-emotional abilities were both predicted by the individual edges comprising the network and associated with the average whole-network strength implying this relationship is observed both at the micro and macro scale properties of the network.

Specifically, on a whole-brain level, associations with outcomes were derived by some optimal balance between positive and negative structural covariance, with stronger positive elements, as compared to stronger negative elements, resulting in better language and social-emotional skills in infanthood. This idea that optimal brain functioning is reliant on a delicate equilibrium of opposing powers is a common theme in neuroscience research especially cortical excitation and inhibition balances (Haider et al. 2006). It is however worth noting that the predictive ability (correlation between predicted and observed values) was relatively modest. Also, the variance explained by 
whole-network strength ranged between $2 \%-4 \%$, similar to previous estimates of the contribution of MRI features in explaining cognitive development in a mixed sample of term and preterm neonates (Girault et al. 2019a), but lower than estimates reported in preterm infants only (Ball et al. 2015). This reinforces other factors such that post-natal development, experience and environment are extremely important (Iso et al. 2007; Koo et al. 2003), that there is variability at the stage of brain development at 18 months and that measures of cognition and outcome are imperfectly measured.

Predictive networks included 1\%-3\% of edges, consistent with other studies utilizing CPM (Rosenberg et al. 2016; Suo et al. 2020; Yip et al. 2019). The resulting predictive networks for language and social-emotional features encompassed the entire cortex, with predictive edges observed both within functional clusters and between functional clusters revealing both local and distributed networks. There was some overlap between predictive networks for social-emotional and language scores. This is not surprising as these measures are conceptually related (i.e., language is critical for social communication) and correlated $(r=-0.52)$. It is not only that producing and understanding speech is critical to communicating with others but it is also postulated that social interaction and social learning experiences are crucial for the proper development of language skills (Kuhl, 2007). Nonetheless, the overlap in predictive networks observed here reached a maximum of $26 \%$, suggesting that while these scores are correlated, they also represent additional behavioural phenotypes and mechanisms.

A large Australian study (Reilly et al. 2007) found that risk factors for developmental language delays such as gender, prematurity and birth weight, birth order, socioeconomic status, maternal mental health and education, and family history of language difficulties explain a small amount of variability in language abilities at age two (up to 7\% of variance). Moreover, the authors found that the most predictive factor of language performance at 12 months is language performance at 8 months and that performance at 24 months is best predicted by abilities at 12 months (Reilly et al. 2006, 2007), thus indicating early trait stability. These findings support our observations that the brain basis for early capabilities begins to shape in very early life, supporting a significant role for genetic and intrauterine 
factors that are further influenced over post-natal development. Moreover, this shows how predicting developmental outcomes from known risk factors has very little power.

Our results point to specific involvement of occipital-parietal, somatosensory-auditory and insularmedial frontal regions. This resonates with established trajectories of brain development, whereby synapses, dendritic growth and myelination are first established in primary cortical regions (Huttenlocher \& Dabholkar, 1997), with cortical grey matter following a lower- to higher-order regional developmental (Gogtay et al. 2004). All of the above-mentioned regions are required for language, communication and social behaviours (Demonet et al. 2005; Porcelli et al. 2019). These larger cortical divisions include specific brain regions traditionally associated with language, such as Broca's area (within the insular and medial/inferior frontal cluster) and the supramarginal gyrus (as part of the occipital and parietal cluster), which has been implicated in studies of toddlers with developmental language disorders using MRI (Morgan et al. 2016). Moreover, the contribution of the cortical primary motor areas complements the association between motor skills and language in both ASC and the general population (Bedford et al. 2016; Gonzalez et al. 2019), whereby better motor development is related to better language development, revealing a close relationship between these seemingly 'independent' domains.

Problems with social functioning and communication are associated with a variety of neurological and psychiatric conditions, most notably ASC. Research on adolescents and adults suggests that social behaviour is reliant on the proper structure and function of stand-alone brain regions, as well as of brain-wide networks (e.g. 'amygdala network', 'empathy network'). Structures identified include but not limited to the temporo-parietal junction, prefrontal cortex, superior temporal gyrus, and the amygdala (Kennedy \& Adolphs, 2012). From a network perspective, in young children with ASC, grey matter covariance showed patterns of decreased connectivity in the cortex that also predicted communication scores (He et al. 2021). Nodal efficiency of tractography networks in infants who were later diagnosed with ASC was also found to be reduced in primary somatosensory, auditory and 
language areas (Lewis et al. 2017), where changes were detected as early as 6 months and related to the level of autistic symptoms at 24 months.

In previous work with a smaller sample size and the inclusion of both term and preterm neonates, cortical FA at birth was found to significantly predict cognitive and language scores at two years using support vector regression (Ouyang et al. 2020) with language-related features including the inferior frontal gyrus, insula and post-central gyrus, regions that were also identified in this current work. A longitudinal study of 33 term-born infants examined the relationship between deformationbased surface distance at neonatal timepoint and the Bayley-III scores at four different time points in the first two years of life (Spann et al. 2014). The authors found significant associations between neonatal volume changes and motor and cognitive scores at 6,12,18 and 24 months, an association we were not able to replicate in this current work. In that study, associations with language scores were also found across all time points, highlighting the cingulate and posterior parietal areas. Interestingly, at 18 months this relationship was weaker compared to performance at 12 or 24 months.

Neonatal MSNs were not predictive or associated with cognitive and motor outcomes. It is possible that cortical structure covariance at birth does not show noticeable cognitive-related variability because as compared to other more 'basic' functions, cognitive skills and associated cortical regions develop later than primary sensory regions. While the neonatal timepoint might be too early to detect cognitive brain correlates, 6-month or 12-month scans might have revealed different results. Nonetheless, it is worth mentioning that Girault et al. (2019) were successful in predicting cognitive ability at age two from white matter connectivity at birth. Lack of results for the motor domain may relate to a narrower spread of performance in motor scores: As can be observed from figure 6, the variability in motor scores, especially in the composite motor scores, is smaller compared to other outcome measures and could influence the ability to detect any meaningful differences. 


\subsection{Limitations}

As a limitation we should highlight that the brain-behaviour associations reported here exclude subcortical structures and the cerebellum as structural features were specifically focused on the cortical surface. CPM has its own limitations as outlined in (Shen et al., 2017), for example, modelling only a linear relationship between variables. However it does provides a clear data-driven framework for implementation and interpretation of behaviour prediction models based on connectivity. We were not able to perform out-of-sample cross-validation for prediction results, only a within-sample validation and as such it remains unclear whether identical results would emerge in a different neonatal sample.

When interpreting scores from developmental assessments, such as the ones used in this study, one should understand their nature: One assessment at 18 months is only a transient screenshot of infant development and is not necessarily indicative of future difficulties at the individual level. Both language delays and the appearance of social-emotional difficulties at an early age do not inevitably mean the continuation of language difficulties or a later autism diagnosis. In addition, these features or delays might not be evident at 18 months but only emerge at a later age. Therefore, any interpretation of infant assessments in the context of future functionality should be done with caution and preferably include follow up at preschool and school-age (Duff et al. 2015; Fountain et al. 2012; Waizbard-Bartov et al. 2021).

\subsection{Conclusions}

In this work we showed that multi-feature multi-modal cortical similarity at birth represented by MSNs are predictive of language and social-emotional abilities in a large group of infants. Cortical regions involved were widespread, with predictive features included connectivity within and across functional cortical domains. Earlier developing cortical regions seemed to be specifically important in this context and overlapped with known language-related regions. This work supports the use of neonatal cortical profiles for means of early detection and support of developmental difficulties. 


\section{Declaration of Competing Interest}

The authors declare no competing interests.

\section{$\underline{\text { Acknowledgements }}$}

We would like to thank the infants and parents who contributed their time to this research and the research team at the Newborn Imaging Centre at Evelina London.

\section{Funding}

The developing Human Connectome Project was funded by the European Research Council under the European Union Seventh Framework Programme (FP/20072013, grant 319456). Infrastructure support was provided by the National Institute for Health Research (NIHR) Mental Health Biomedical Research Centre at South London, Maudsley NHS Foundation Trust, King's College London and the NIHR Mental Health Biomedical Research Centre at Guy's and St Thomas' Hospitals NHS Foundation Trust. The views expressed are those of the author(s) and not necessarily those of the NHS, the NIHR or the Department of Health and Social Care. The study was supported in part by the Wellcome Engineering and Physical Sciences Research Council Centre for Medical Engineering at King's College London (grant WT 203148/Z/16/Z) and the Medical Research Council (UK) (grants MR/K006355/1, MR/LO11530/1). D.F's PhD is supported by the Sackler Institute for Translational Neurodevelopment at King's College London and the Medical Research Council Centre for Neurodevelopmental Disorders, King's College London. J.O.M. is supported by a Sir Henry Dale Fellowship jointly funded by the Wellcome Trust and the Royal Society (grant 206675/Z/17/Z). J.O.M., D.E. and G.M. received support from the Medical Research Council Centre for Neurodevelopmental Disorders, King's College London (grant MR/N026063/1). D.C. is supported by the Flemish Research Foundation (FWO; fellowship no. 12ZV420N). 
Alexander-Bloch, A., Giedd, J., \& Bullmore, E. (2013a). Imaging structural co-variance between human brain regions. Nat Rev Neurosci, 14, 322-336. doi:10.1038/nrn3465

Alexander-Bloch, A., Raznahan, A., Bullmore, E., \& Giedd, J. (2013b). The Convergence of Maturational Change and Structural Covariance in Human Cortical Networks. Journal of Neuroscience, 33(7), 2889-2899. doi:10.1523/JNEUROSCI.3554-12.2013

Allison, C., Baron-Cohen, S., Wheelwright, S., Charman, T., Richler, J., Pasco, G., \& Brayne, C. (2008). The Q-CHAT (Quantitative CHecklist for Autism in Toddlers): a normally distributed quantitative measure of autistic traits at 18-24 months of age: preliminary report. J Autism Dev Disord, 38(8), 1414-1425. doi:10.1007/s10803-007-0509-7

Andersson, J. L. R., Skare, S., \& Ashburner, J. (2003). How to correct susceptibility distortions in spin-echo echo-planar images: Application to diffusion tensor imaging. Neuroimage, 20(2), 870-888. doi:10.1016/S1053-8119(03)00336-7

Aylward, G. P. (2014). Neurodevelopmental Outcomes of Infants Born Prematurely. Journal of Developmental \& Behavioral Pediatrics, 35(6). doi: 10.1097/00004703-200512000-00008

Ball, G., Pazderova, L., Chew, A., Tusor, N., Merchant, N., Arichi, T., . . Counsell, S. J. (2015). Thalamocortical Connectivity Predicts Cognition in Children Born Preterm. Cereb Cortex, 25(11), 4310-4318. doi:10.1093/cercor/bhu331

Ball, G., Seidlitz, J., O’Muircheartaigh, J., Dimitrova, R., Fenchel, D., Makropoulos, A., . . Hutter, J. (2020). Cortical morphology at birth reflects spatiotemporal patterns of gene expression in the fetal human brain. PLoS biology, 18(11), e3000976. doi: 10.1371/journal.pbio.3000976

Batalle, D., Edwards, A. D., \& O'Muircheartaigh, J. (2018). Annual Research Review: Not just a small adult brain: understanding later neurodevelopment through imaging the neonatal brain. J Child Psychol Psychiatry, 59(4), 350-371. doi:10.1111/jcpp.12838

Bayley, N. (2006). Bayley Scales of Infant and Toddler Development-Third Edition. San Antonio, Tx: Harcourt Assessment.

Bedford, R., Pickles, A., \& Lord, C. (2016). Early gross motor skills predict the subsequent development of language in children with autism spectrum disorder. Autism Res, 9(9), 993-1001. doi:10.1002/aur.1587

Bozek, J., Makropoulos, A., Schuh, A., Fitzgibbon, S., Wright, R., Glasser, M. F., . . Robinson, E. C. (2018). Construction of a neonatal cortical surface atlas using Multimodal Surface Matching in the Developing Human Connectome Project. Neuroimage, 179, 11-29.

doi:10.1016/j.neuroimage.2018.06.018

Christiaens, D., Cordero-Grande, L., Pietsch, M., Hutter, J., Price, A. N., Hughes, E. J., . . Tournier, J. D. (2021). Scattered slice SHARD reconstruction for motion correction in multi-shell diffusion MRI. Neuroimage, 225, 117437. doi:10.1016/j.neuroimage.2020.117437 
Cooper, G. M., Coe, B. P., Girirajan, S., Rosenfeld, J. A., Vu, T. H., Baker, C., . . Eichler, E. E. (2011). A copy number variation morbidity map of developmental delay. Nat Genet, 43(9), 838-846. doi:10.1038/ng.909

Cordero-Grande, L., Hughes, E. J., Hutter, J., Price, A. N., \& Hajnal, J. V. (2018). Three-dimensional motion corrected sensitivity encoding reconstruction for multi-shot multi-slice MRI: Application to neonatal brain imaging. Magnetic Resonance in Medicine, 79(3), 1365-1376. doi:10.1002/mrm.26796 Craney, T. A., \& Surles, J. G. (2002). Model-Dependent Variance Inflation Factor Cutoff Values. Quality Engineering, 14(3), 391-403. doi:10.1081/QEN-120001878

Dawson, G., Rogers, S., Munson, J., Smith, M., Winter, J., Greenson, J., . . Varley, J. (2010). Randomized, controlled trial of an intervention for toddlers with autism: the Early Start Denver Model. Pediatrics, 125(1), e17-23. doi:10.1542/peds.2009-0958

Demonet, J. F., Thierry, G., \& Cardebat, D. (2005). Renewal of the neurophysiology of language: functional neuroimaging. Physiol Rev, 85(1), 49-95. doi:10.1152/physrev.00049.2003

Duff, F. J., Nation, K., Plunkett, K., \& Bishop, D. (2015). Early prediction of language and literacy problems: is 18 months too early? PeerJ, 3, e1098. doi:10.7717/peerj.1098

Fenchel, D., Dimitrova, R., Seidlitz, J., Robinson, E. C., Batalle, D., Hutter, J., . . O'Muircheartaigh, J. (2020). Development of Microstructural and Morphological Cortical Profiles in the Neonatal Brain. Cereb Cortex, 30(11), 5767-5779. doi:10.1093/cercor/bhaa150

Fenson, L., Dale, P. S., Reznick, J. S., Bates, E., Thal, D. J., \& Pethick, S. J. (1994). Variability in early communicative development. Monogr Soc Res Child Dev, 59(5), 1-173; discussion 174-185. Flensborg-Madsen, T., \& Mortensen, E. L. (2018). Developmental milestones during the first three years as precursors of adult intelligence. Dev Psychol, 54(8), 1434-1444. doi:10.1037/dev0000545 Fountain, C., Winter, A. S., \& Bearman, P. S. (2012). Six developmental trajectories characterize children with autism. Pediatrics, 129(5), e1112-1120. doi:10.1542/peds.2011-1601

Girault, J. B., Cornea, E., Goldman, B. D., Knickmeyer, R. C., Styner, M., \& Gilmore, J. H. (2019a). White matter microstructural development and cognitive ability in the first 2 years of life. Hum Brain Mapp, 40(4), 1195-1210. doi:10.1002/hbm.24439

Girault, J. B., Munsell, B. C., Puechmaille, D., Goldman, B. D., Prieto, J. C., Styner, M., \& Gilmore, J. H. (2019b). White matter connectomes at birth accurately predict cognitive abilities at age 2 .

Neuroimage, 192, 145-155. doi:10.1016/j.neuroimage.2019.02.060

Gogtay, N., Giedd, J. N., Lusk, L., Hayashi, K. M., Greenstein, D., Vaituzis, A. C., . . Thompson, P. M. (2004). Dynamic mapping of human cortical development during childhood through early adulthood. Proc Natl Acad Sci U S A, 101(21), 8174-8179. doi:10.1073/pnas.0402680101 Gonzalez, S. L., Alvarez, V., \& Nelson, E. L. (2019). Do Gross and Fine Motor Skills Differentially Contribute to Language Outcomes? A Systematic Review. Front Psychol, 10, 2670.

doi:10.3389/fpsyg.2019.02670 
Gurevitz, M., Geva, R., Varon, M., \& Leitner, Y. (2014). Early markers in infants and toddlers for development of ADHD. J Atten Disord, 18(1), 14-22. doi:10.1177/1087054712447858

Haider, B., Duque, A., Hasenstaub, A. R., \& McCormick, D. A. (2006). Neocortical network activity in vivo is generated through a dynamic balance of excitation and inhibition. J Neurosci, 26(17), 45354545. doi:10.1523/JNEUROSCI.5297-05.2006

He, C., Cortes, J. M., Kang, X., Cao, J., Chen, H., Guo, X., . . Duan, X. (2021). Individual-based morphological brain network organization and its association with autistic symptoms in young children with autism spectrum disorder. Hum Brain Mapp. doi:10.1002/hbm.25434

Hughes, E. J., Winchman, T., Padormo, F., Teixeira, R., Wurie, J., Sharma, M., . . Hajnal, J. V. (2017). A dedicated neonatal brain imaging system. Magn Reson Med, 78(2), 794-804.

doi:10.1002/mrm.26462

Huttenlocher, P. R., \& Dabholkar, A. S. (1997). Regional differences in synaptogenesis in human cerebral cortex. J Comp Neurol, 387(2), 167-178. doi:10.1002/(sici)1096-

9861(19971020)387:2<167::aid-cne1>3.0.co;2-z

Hutter, J., Tournier, J. D., Price, A. N., Cordero-Grande, L., Hughes, E. J., Malik, S., . . Hajnal, J. V. (2018). Time-efficient and flexible design of optimized multishell HARDI diffusion. Magn Reson Med, 79(3), 1276-1292. doi:10.1002/mrm.26765

Iso, H., Simoda, S., \& Matsuyama, T. (2007). Environmental change during postnatal development alters behaviour, cognitions and neurogenesis of mice. Behav Brain Res, 179(1), 90-98.

doi:10.1016/j.bbr.2007.01.025

Jeong, J., Franchett, E. E., Ramos de Oliveira, C. V., Rehmani, K., \& Yousafzai, A. K. (2021).

Parenting interventions to promote early child development in the first three years of life: A global systematic review and meta-analysis. PLoS Med, 18(5), e1003602.

doi:10.1371/journal.pmed.1003602

Kellner, E., Dhital, B., Kiselev, V. G., \& Reisert, M. (2016). Gibbs-ringing artifact removal based on local subvoxel-shifts. Magn Reson Med, 76(5), 1574-1581. doi:10.1002/mrm.26054

Kennedy, D. P., \& Adolphs, R. (2012). The social brain in psychiatric and neurological disorders.

Trends Cogn Sci, 16(11), 559-572. doi:10.1016/j.tics.2012.09.006

Keunen, K., Benders, M. J., Leemans, A., Fieret-Van Stam, P. C., Scholtens, L. H., Viergever, M. A., . . van den Heuvel, M. P. (2017). White matter maturation in the neonatal brain is predictive of school age cognitive capacities in children born very preterm. Dev Med Child Neurol, 59(9), 939-946. doi:10.1111/dmen.13487

Koo, J. W., Park, C. H., Choi, S. H., Kim, N. J., Kim, H. S., Choe, J. C., \& Suh, Y. H. (2003). The postnatal environment can counteract prenatal effects on cognitive ability, cell proliferation, and synaptic protein expression. FASEB J, 17(11), 1556-1558. doi:10.1096/fj.02-1032fje

Kuhl, P. K. (2007). Is speech learning 'gated' by the social brain? Dev Sci, 10(1), 110-120. doi:10.1111/j.1467-7687.2007.00572.x 
Landa, R., \& Garrett-Mayer, E. (2006). Development in infants with autism spectrum disorders: a prospective study. J Child Psychol Psychiatry, 47(6), 629-638. doi:10.1111/j.1469-7610.2006.01531.x Lewis, J. D., Evans, A. C., Pruett, J. R., Jr., Botteron, K. N., McKinstry, R. C., Zwaigenbaum, L., . . . Infant Brain Imaging Study, N. (2017). The Emergence of Network Inefficiencies in Infants With Autism Spectrum Disorder. Biol Psychiatry, 82(3), 176-185. doi:10.1016/j.biopsych.2017.03.006 Liu, S., Cai, W., Liu, S., Zhang, F., Fulham, M., Feng, D., . . Kikinis, R. (2015). Multimodal neuroimaging computing: a review of the applications in neuropsychiatric disorders. Brain Inform, 2(3), 167-180. doi:10.1007/s40708-015-0019-x

Makropoulos, A., Robinson, E. C., Schuh, A., Wright, R., Fitzgibbon, S., Bozek, J., . . Rueckert, D. (2018). The developing human connectome project: A minimal processing pipeline for neonatal cortical surface reconstruction. Neuroimage, 173, 88-112. doi:10.1016/j.neuroimage.2018.01.054 Marshall, C. R., Noor, A., Vincent, J. B., Lionel, A. C., Feuk, L., Skaug, J., . . Scherer, S. W. (2008). Structural variation of chromosomes in autism spectrum disorder. Am J Hum Genet, 82(2), 477-488. doi:10.1016/j.ajhg.2007.12.009

Morgan, A., Bonthrone, A., \& Liegeois, F. J. (2016). Brain basis of childhood speech and language disorders: are we closer to clinically meaningful MRI markers? Curr Opin Pediatr, 28(6), 725-730. doi:10.1097/MOP.0000000000000420

Morgan, S. E., Seidlitz, J., Whitaker, K. J., Romero-Garcia, R., Clifton, N. E., Scarpazza, C., . . Bullmore, E. T. (2019). Cortical patterning of abnormal morphometric similarity in psychosis is associated with brain expression of schizophrenia-related genes. Proc Natl Acad Sci U S A, 116(19), 9604-9609. doi:10.1073/pnas.1820754116

Murray, G. K., Jones, P. B., Kuh, D., \& Richards, M. (2007). Infant developmental milestones and subsequent cognitive function. Ann Neurol, 62(2), 128-136. doi:10.1002/ana.21120

Mwaniki, M. K., Atieno, M., Lawn, J. E., \& Newton, C. R. (2012). Long-term neurodevelopmental outcomes after intrauterine and neonatal insults: a systematic review. Lancet, 379(9814), 445-452. doi:10.1016/S0140-6736(11)61577-8

Ouyang, M., Peng, Q., Jeon, T., Heyne, R., Chalak, L., \& Huang, H. (2020). Diffusion-MRI-based regional cortical microstructure at birth for predicting neurodevelopmental outcomes of 2-year-olds. Elife, 9. doi:10.7554/eLife.58116

Ozonoff, S., Young, G. S., Carter, A., Messinger, D., Yirmiya, N., Zwaigenbaum, L., . . Stone, W. L. (2011). Recurrence risk for autism spectrum disorders: a Baby Siblings Research Consortium study. Pediatrics, 128(3), e488-495. doi:10.1542/peds.2010-2825

Pascal, A., Govaert, P., Oostra, A., Naulaers, G., Ortibus, E., \& Van den Broeck, C. (2018).

Neurodevelopmental outcome in very preterm and very-low-birthweight infants born over the past decade: a meta-analytic review. Dev Med Child Neurol, 60(4), 342-355. doi:10.1111/dmcn.13675

Piek, J. P. (2002). The role of variability in early motor development. Infant Behav Dev, 25(4), 452465. doi:https://doi.org/10.1016/S0163-6383(02)00145-5 
Porcelli, S., Van Der Wee, N., van der Werff, S., Aghajani, M., Glennon, J. C., van Heukelum, S., . . . Serretti, A. (2019). Social brain, social dysfunction and social withdrawal. Neurosci Biobehav Rev, 97, 10-33. doi:10.1016/j.neubiorev.2018.09.012

Reh, R. K., Dias, B. G., Nelson, C. A., 3rd, Kaufer, D., Werker, J. F., Kolb, B., . . Hensch, T. K. (2020). Critical period regulation across multiple timescales. Proc Natl Acad Sci U S A, 117(38), 23242-23251. doi:10.1073/pnas.1820836117

Reilly, S., Eadie, P., Bavin, E. L., Wake, M., Prior, M., Williams, J., . . Ukoumunne, O. C. (2006). Growth of infant communication between 8 and 12 months: a population study. J Paediatr Child Health, 42(12), 764-770. doi:10.1111/j.1440-1754.2006.00974.x

Reilly, S., Wake, M., Bavin, E. L., Prior, M., Williams, J., Bretherton, L., . . Ukoumunne, O. C. (2007). Predicting language at 2 years of age: a prospective community study. Pediatrics, 120(6), e1441-1449. doi:10.1542/peds.2007-0045

Riva, V., Caruso, A., Apicella, F., Valeri, G., Vicari, S., Molteni, M., \& Scattoni, M. L. (2021). Early developmental trajectories of expressive vocabulary and gesture production in a longitudinal cohort of Italian infants at high-risk for Autism Spectrum Disorder. Autism Res 14(7), 421-1433.

doi:10.1002/aur.2493

Robinson, E. C., Jbabdi, S., Andersson, J., Smith, S., Glasser, M. F., Van Essen, D. C., . . Jenkinson, M. (2013). Multimodal surface matching: Fast and generalisable cortical registration using discrete optimisation. In Gee J. C., Joshi S., Pohl K. M., Wells W. M., Zöllei L. (eds) Information Processing in Medical Imaging. IPMI 2013. Lecture Notes in Computer Science, 7917, 475-486: Springer, Berlin, Heidelberg. doi: 10.1007/978-3-642-38868-2_40

Robinson, E. C., Jbabdi, S., Glasser, M. F., Andersson, J., Burgess, G. C., Harms, M. P., . . Jenkinson, M. (2014). MSM: A new flexible framework for multimodal surface matching. Neuroimage, 100, 414-426. doi:10.1016/j.neuroimage.2014.05.069

Rosenberg, M. D., Casey, B. J., \& Holmes, A. J. (2018). Prediction complements explanation in understanding the developing brain. Nat Commun, 9(1), 589. doi:10.1038/s41467-018-02887-9 Rosenberg, M. D., Finn, E. S., Scheinost, D., Papademetris, X., Shen, X., Constable, R. T., \& Chun, M. M. (2016). A neuromarker of sustained attention from whole-brain functional connectivity. Nat Neurosci, 19(1), 165-171. doi:10.1038/nn.4179

Rosenberg, M. D., Hsu, W.-T., Scheinost, D., Todd Constable, R., \& Chun, M. M. (2018).

Connectome-based Models Predict Separable Components of Attention in Novel Individuals. Journal of Cognitive Neuroscience, 30(2), 160-173. doi:10.1162/jocn_a_01197

Rosenberg, S. A., Zhang, D., \& Robinson, C. C. (2008). Prevalence of developmental delays and participation in early intervention services for young children. Pediatrics, 121(6), e1503-1509. doi:10.1542/peds.2007-1680 
Seidlitz, J., Nadig, A., Liu, S., Bethlehem, R. A. I., Vertes, P. E., Morgan, S. E., . . Raznahan, A. (2020). Transcriptomic and cellular decoding of regional brain vulnerability to neurogenetic disorders. Nat Commun, 11(1), 3358. doi:10.1038/s41467-020-17051-5

Seidlitz, J., Váša, F., Shinn, M., Romero-Garcia, R., Whitaker, K. J., Vértes, P. E., . . Bullmore, E. T. (2018). Morphometric Similarity Networks Detect Microscale Cortical Organization and Predict Inter-Individual Cognitive Variation. Neuron, 97(1), 231-247.e237. doi:10.1016/j.neuron.2017.11.039 Shahat, A. R. S., \& Greco, G. (2021). The Economic Costs of Childhood Disability: A Literature Review. Int J Environ Res Public Health, 18(7). doi:10.3390/ijerph18073531

Shen, X., Finn, E. S., Scheinost, D., Rosenberg, M. D., Chun, M. M., Papademetris, X., \& Constable, R. T. (2017). Using connectome-based predictive modeling to predict individual behavior from brain connectivity. Nat Protoc, 12(3), 506-518. doi:10.1038/nprot.2016.178

Shivers, C. M., Jackson, J. B., \& McGregor, C. M. (2019). Functioning Among Typically Developing Siblings of Individuals with Autism Spectrum Disorder: A Meta-Analysis. Clin Child Fam Psychol Rev, 22(2), 172-196. doi:10.1007/s10567-018-0269-2

Sorensen, H. J., Mortensen, E. L., Schiffman, J., Reinisch, J. M., Maeda, J., \& Mednick, S. A. (2010). Early developmental milestones and risk of schizophrenia: a 45-year follow-up of the Copenhagen Perinatal Cohort. Schizophr Res, 118(1-3), 41-47. doi:10.1016/j.schres.2010.01.029

Spann, M. N., Bansal, R., Rosen, T. S., \& Peterson, B. S. (2014). Morphological features of the neonatal brain support development of subsequent cognitive, language, and motor abilities. Hum Brain Mapp, 35(9), 4459-4474. doi:10.1002/hbm.22487

Stabile, M., \& Allin, S. (2012). The economic costs of childhood disability. Future Child, 22(1), 6596. doi:10.1353/foc. 2012.0008

Stromswold, K. (1998). Genetics of spoken language disorders. Hum Biol, 70(2), 297-324. Suo, X., Lei, D., Li, W., Yang, J., Li, L., Sweeney, J. A., \& Gong, Q. (2020). Individualized Prediction of PTSD Symptom Severity in Trauma Survivors From Whole-Brain Resting-State Functional Connectivity. Front Behav Neurosci, 14, 563152. doi:10.3389/fnbeh.2020.563152 Tournier, J. D., Christiaens, D., Hutter, J., Price, A. N., Cordero $\square$ Grande, L., Hughes, E., . . Hajnal, J. V. (2020). A data $\square$ driven approach to optimising the encoding for multi $\square$ shell diffusion MRI with application to neonatal imaging. NMR Biomed, 33(9), e4348. doi:10.1002/nbm.4348.

Tournier, J. D., Smith, R., Raffelt, D., Tabbara, R., Dhollander, T., Pietsch, M., . . Connelly, A. (2019). MRtrix3: A fast, flexible and open software framework for medical image processing and visualisation. Neuroimage, 15 (202), 116137-116137. doi: 10.1016/j.neuroimage.2019.116137. Tulay, E. E., Metin, B., Tarhan, N., \& Arikan, M. K. (2019). Multimodal Neuroimaging: Basic Concepts and Classification of Neuropsychiatric Diseases. Clin EEG Neurosci, 50(1), 20-33. doi:10.1177/1550059418782093

Van't Hooft, J., van der Lee, J. H., Opmeer, B. C., Aarnoudse-Moens, C. S., Leenders, A. G., Mol, B. W., \& de Haan, T. R. (2015). Predicting developmental outcomes in premature infants by term 
equivalent MRI: systematic review and meta-analysis. Syst Rev, 4, 71. doi:10.1186/s13643-015-00587

Veraart, J., Novikov, D. S., Christiaens, D., Ades-aron, B., Sijbers, J., \& Fieremans, E. (2016).

Denoising of diffusion MRI using random matrix theory. Neuroimage, 142, 394-406.

doi:10.1016/j.neuroimage.2016.08.016

Waizbard-Bartov, E., Ferrer, E., Young, G. S., Heath, B., Rogers, S., Wu Nordahl, C., . . Amaral, D.

G. (2021). Trajectories of Autism Symptom Severity Change During Early Childhood. J Autism Dev

Disord, 51(1), 227-242. doi:10.1007/s10803-020-04526-Z

Wee, C. Y., Tuan, T. A., Broekman, B. F., Ong, M. Y., Chong, Y. S., Kwek, K., . . Qiu, A. (2017).

Neonatal neural networks predict children behavioral profiles later in life. Hum Brain Mapp, 38(3),

1362-1373. doi:10.1002/hbm.23459

Yip, S. W., Scheinost, D., Potenza, M. N., \& Carroll, K. M. (2019). Connectome-Based Prediction of Cocaine Abstinence. Am J Psychiatry, 176(2), 156-164. doi:10.1176/appi.ajp.2018.17101147

Zhang, H., Schneider, T., Wheeler-Kingshott, C. A., \& Alexander, D. C. (2012). NODDI: Practical in vivo neurite orientation dispersion and density imaging of the human brain. Neuroimage, 61(4), 1000-

1016. doi:10.1016/J.NEUROIMAGE.2012.03.072 